\title{
Promoción de ciudades Patrimonio de la Humanidad: estudio sobre la traducción del léxico relacionado con la gastronomía
}

\author{
Promotion of World Heritage Cities: A study \\ on the translation of the lexicon related to gastronomy \\ Eva M. Mestre-Mestre \& Teresa Molés-Cases
}

Universitat Politècnica de València. evamestre@upvnet.upv.es, temoca1@upv.es Recibido: 17.04.2019. Aceptado: 02.07.2019

Resumen: Esta contribución presenta un estudio de traducción español-inglés basado en un corpus formado por las páginas web turísticas oficiales de las 15 ciudades españolas que hasta la fecha han sido declaradas Patrimonio Cultural Inmaterial de la Humanidad de la UNESCO. Nos centramos en la traducción de los referentes culturales y, más concretamente, en el léxico relacionado con la comida y la bebida. Las técnicas de traducción observadas se agrupan siguiendo propuestas de clasificación existentes en la literatura adaptadas a los referentes culturales. Los resultados obtenidos permiten llegar a conclusiones en cuanto a las tendencias de traducción (extranjerizantes o familiarizantes) en este contexto y destacan la urgente necesidad de valorar y recurrir a la traducción profesional en el sector del turismo.

Palabras clave: traducción español-inglés; referentes culturales; turismo; lenguaje para fines específicos; técnicas de traducción.

\begin{abstract}
This contribution presents a Spanish-English translation study of a corpus composed of the official tourism web sites of the 15 Spanish cities declared as UNESCO World Heritage Cities to date. Here, attention will be paid to the translation of cultural references, and more specifically, lexicon related to food and beverages. The translation techniques observed are analysed following existing classifications that are adapted to cultural references. The results obtained allow drawing conclusions regarding (foreignizing or domesticating) translation tendencies in this context and highlight the urgent need to acknowledge the value and resort to professional translation in the field of tourism.
\end{abstract}

Keywords: Spanish-English translation; cultural references; tourism; language for specific purposes; translation techniques.

》) Mestre-Mestre, Eva M. \& Molés-Cases, Teresa. 2019. "Promoción de ciudades Patrimonio de la Humanidad: estudio sobre la traducción del léxico relacionado con la gastronomía”. Quaderns de Filologia: Estudis Lingüístics XXIV: 329-347. doi: 10.7203/QF.24.16315 



\section{Introducción}

Aunque el turismo es uno de los principales motores económicos de España, de vez en cuando todavía salen a la palestra casos de traducción no profesional (realizada, generalmente, a partir del traductor de Google) en este sector. Uno de los escándalos más sonados y recientes en este sentido es la traducción literal del conocido Centro Botín por la expresión inglesa "Loot Center" ("Centro del Saqueo") en la página web de turismo oficial de la ciudad de Santander con motivo de la edición del año 2018 de FITUR (Feria Internacional de Turismo), un evento precisamente destinado a la internacionalización. Otro ejemplo similar relacionado con la promoción turística es el que se publicaba en 2015 en la página web del municipio gallego de As Pontes (Galicia), donde se invitaba a disfrutar de la gastronomía en la "Feria del clítoris" y no en la "Feria de grelos", por haber confundido el traductor el grelo gallego (verdura típica de Galicia) con el vulgarismo portugués a la hora de traducir al castellano. Estos ejemplos, que a primera vista pueden parecer simples anécdotas, no hacen sino dañar la imagen de quien publica las páginas en particular y la del sector turístico en general.

La traducción turística es un tipo de traducción especializada donde la transferencia cultural juega un papel clave, en tanto que la prioridad es presentar y promocionar la cultura origen en el contexto de la cultura meta. Nuestro interés por ahondar en esta cuestión nos llevó a analizar la traducción al inglés de las páginas web oficiales de las ciudades españolas que hasta la fecha han sido declaradas Patrimonio Cultural Inmaterial de la Humanidad de la UNESCO e identificar las técnicas de traducción observadas en el caso de los referentes culturales relacionados con la comida y la bebida. La gastronomía se considera cada vez más como parte del patrimonio que se transmite. No solamente a nivel social y económico, sino también cultural y, en última instancia, sobre todo turístico. Es uno de los principales atractivos que explotan las ciudades para darse a conocer y atraer turistas ávidos de nuevas experiencias y autenticidad.

La estructura del artículo es la que sigue: en primer lugar, realizaremos una breve revisión teórica en torno a la traducción especializada (concretamente, del sector turístico) y las técnicas de traducción de los referentes culturales; a continuación, presentaremos la metodología aplicada para el análisis; seguidamente se detallarán los resultados, que 
son tanto de corte cualitativo, como cuantitativo; finalmente, presentaremos las conclusiones del estudio y trazaremos líneas de acción futura.

\section{Fundamentos teóricos}

\subsection{La traducción especializada: el sector del turismo}

Kelly (1998) define el texto turístico como:

any text published by a public or private organization of any kind intended a) to give information to any kind of visitor or b) to advertise a destination $[\ldots]$ and encourage visitors to go there (1998: 35).

La traducción de textos turísticos es un tipo de traducción especializada cuya finalidad es doble: informativa y persuasiva. Si bien la traducción de textos turísticos cubre una gran diversidad de tipos textuales (por ejemplo: folletos, guías, menús/cartas), en muchas ocasiones no se le atribuye el valor que merece en un escenario como España, donde tiene una importancia clave, ${ }^{1}$ pero donde todavía existe una actitud negativa generalizada de este mercado hacia la traducción profesional. Así lo afirma Soto Almela (2013):

Podemos incluso afirmar que la traducción de textos turísticos está infravalorada como traducción especializada $\mathrm{y}$, de hecho, la mayoría de traducciones turísticas realizadas en nuestro país producen incomprensión en su lectura y contienen errores lingüísticos, lo que provoca un distanciamiento de los visitantes con respecto a la cultural española (2013: 236).

Asimismo, durante los últimos años se han desarrollado nuevas formas de promocionar el turismo a través de internet, como por ejemplo páginas web o aplicaciones móviles. Si bien la localización se realiza con frecuencia a partir de herramientas de traducción asistida por ordenador, ello no implica que el factor humano sea prescindible, en tanto que la interculturalidad desempeña un papel clave en el ámbito del turismo $\mathrm{y}$ es necesario contar con un agente humano experto que trace una estra-

\footnotetext{
${ }^{1} \mathrm{Y}$ tampoco en otros escenarios internacionales, tal y como indican Neubert y Shreve
} (1992) o Chesterman (1997). 
tegia de traducción coherente con el objeto informativo-persuasivo del texto turístico (en este caso, la página web turística) (cf. Pierini, 2007) y que se responsabilice del producto final para su publicación.

\subsection{Las técnicas de traducción de los referentes culturales}

En los Estudios Descriptivos de Traducción (EDT) (Toury, 1995), el análisis microlingüístico (y más concretamente, la comparación de pares de fragmentos origen y meta) suele explicarse a través del concepto técnica de traducción. En la literatura encontramos una serie de contribuciones que se centran expresamente en las técnicas de traducción del fenómeno de los referentes culturales, por ejemplo: Martínez Sánchez e Íñigo Ros (1998), Newmark (1988), Marco (2004, 2019), Molina y Hurtado (2002), Oster y Molés-Cases (2016), Rodríguez Abella (2008, 2009). A continuación, revisaremos los trabajos de Martínez Sánchez e Íñigo Ros (1998), Rodríguez Abella (2008, 2009), Oster y Molés-Cases (2016) y Marco (2019), ya que analizan específicamente los referentes culturales relacionados con la comida y la bebida como problema de traducción.

Martínez Sánchez e Íñigo Ros (1998) analizan las técnicas de traducción ${ }^{2}$ observadas en la traducción de referentes culturales de la gastronomía (tanto platos típicos, como ingredientes) a partir de un corpus de folletos turísticos en español y sus respectivas traducciones al inglés. Inspiradas por la propuesta de Newmark (1988), estas autoras identifican principalmente las siguientes técnicas: (1) préstamo (cocidos gallegos > cocidos gallegos), (2) calco (denominación de origen > designation of origin), (3) hipónimo (puchero $>$ stew), hiperónimo (potaje $>$ dish), (4) traducción por equivalente cultural (turrón $>$ nougat), (5) omisión (cava $>-$ ), (6) descripción (potaje > vegetable stew), (7) explicación (vinos generosos $>$ full-bodied wines) y (8) combinación

\footnotetext{
${ }^{2}$ Martínez Sánchez e Íñigo Ros (1998) se refieren realmente al concepto estrategia de traducción. No obstante, dado que en la literatura ha habido cierta confusión terminológica en torno a los conceptos estrategia, método, técnica y error, en esta contribución nos limitaremos al uso de técnica de traducción tal y como la entienden Molina y Hurtado (2002). Según estas autoras, las técnicas de traducción sirven para describir "the actual steps taken by the translators in each textual micro-unit," lo que nos ayuda a obtener "clear data about the general methodological option chosen" (Molina y Hurtado, 2002: 49).
} 
de técnicas (por ejemplo, préstamo con explicación: mantecados > mantecados [buns variedly flavoured]). Los resultados del estudio indican que las técnicas de préstamo y traducción por términos culturales equivalentes son las más frecuentes en el corpus. Las autoras justifican parte de los resultados a partir de la función o el propósito del folleto turístico, en tanto que el "color local añadido por los préstamos" presenta una función apelativa y puede atraer a potenciales turistas (1998: 168); asimismo, explican que las convenciones típicas de este tipo de texto (por ejemplo: inclusión de imágenes) pueden complementar la comprensión de términos generales o específicos en la traducción; no obstante, afirman que "en las traducciones estudiadas su carácter informativo (se persigue dar una información fidedigna) y el vocativo (se quiere atraer al turista) entran en conflicto (1998: 169)" (cf. gradación extranjerizante - familiarizante, Venuti, 1995).

Rodríguez Abella (2009) estudia los referentes culturales relacionados con la gastronomía observados en un corpus formado por 12 folletos turísticos de TURESPAÑA y sus respectivas traducciones al italiano. Identifica las siguientes técnicas de traducción: (1) traducción literal (jamones ibéricos > prosicutti iberici “jamones ibéricos"), (2) préstamo puro (gazpacho > gazpacho), (3) descripción (cocina serrana > cucina caratteristica della montagna "cocina característica de la montaña"), (4) adaptación (pistos $>$ peperonate "plato a base de pimientos troceados y cocinados con aceite, cebolla y ajo"), (5) generalización (natillas $>$ crema "crema"), (6) equivalente acuñado (pimentón > paprica), (7) elisión (tapeo $>-$ ), (8) error de traducción (bollo maimón $>$ almendras garrapiñadas [mandorle]). Además de estas técnicas, la autora identifica otras que solo aparecen en (8) combinación (particularización, amplificación, errata, reducción); por ejemplo: préstamo puro y amplificación: ajoarriero > ajoarriero (baccalà all'aglio) "ajoarriero (bacalao al ajo)"; o préstamo puro y errata (cochifrito $>$ cuchifrito). El elevado número de préstamos puros en las traducciones analizadas evidencia la distancia con el lector meta; en otras palabras, las traducciones analizadas estarían muy marcadas culturalmente desde la perspectiva de la cultura origen, de la que son más próximas.

En un trabajo anterior, Rodríguez Abella (2008) analiza los referentes culturales del campo de la gastronomía en el campo literario, concretamente en la traducción al italiano de la novela El hombre de mi vida (Vázquez Montalbán, 2000), donde identifica las técnicas de traducción 
para tres fenómenos (productos/ingredientes, técnicas de cocina/elaboraciones y platos). A continuación se indican las técnicas observadas para productos/ingredientes y platos, ya que son los que interesan a efectos de este artículo: (1) generalización (morro de bacalao $>$ bacalà "bacalao"), (2) particularización (embutidos > salame "salami"), (3) préstamo puro (tapas $>$ tapas), (4) descripción (escudellas $>$ escudellas + nota del traductor), (5) equivalente acuñado (cocas $>$ focacce "pan enriquecido con aceite y sal"), (6) traducción literal (migas con chorizo $>$ pane sminuzzato e fritto con salame icante "pan desmenuzado frito con salami picante"). En el corpus analizado, algunas técnicas (elisión, amplificación, compensación) solo aparecen (7) en combinación; por ejemplo, elisión y traducción literal (empanada de bonito en hojaldre $>$ tonno in millefoglie "atún en milhojas"). Dado que la generalización es la técnica más recurrente en el corpus, el análisis de los datos permite concluir que, en este caso, la traductora ha optado por una traducción en general no marcada culturalmente.

En Oster y Molés-Cases (2016) se presenta un estudio sobre las dificultades de traducción en el caso del léxico relacionado con la comida y la bebida (concretamente: referentes culturales, acciones/maneras de comer y beber y expresiones metafóricas que adoptan comida o bebida como dominio fuente). El corpus analizado se corresponde con el subcorpus alemán>castellano/catalán del corpus literario COVALT. ${ }^{3}$ Para el caso concreto de los referentes culturales, que es el que interesa a efectos de este artículo, se identifican las siguientes técnicas de traducción (cf. gradación extranjerizante - familiarizante, Venuti, 1995): (1) adaptación intercultural (Gemüse-Eintopf "sopa o guiso de verduras" $>$ menestra de verduras), (2) adaptación intracultural (Lebkuchenmann "pan de especias típico de Navidad" > pa de gingebre), (3) generalización (Butterbrot "pan con mantequilla" > pan), (4) descripción (Negerkuss "dulce tradicional con merengue y chocolate" > bola de chocolate rellena de nata), (5) omisión (Gummibärchen "ositos de goma" $>i$ d'altres llepolies), (6) recreación (Spekulatius "galletas de especias típicas de Navidad" > pasta de pastaflora) y (7) traducción literal

\footnotetext{
${ }^{3}$ El corpus COVALT (Corpus Valenciano de Literatura Traducida) es un corpus compilado en la Universitat Jaume I (http://www.covalt.uji.es) formado por textos narrativos que incluye varios componentes: a) textos originales en inglés, francés y alemán, b) sus respectivas traducciones al catalán y al castellano y c) textos originales (comparables) en catalán y castellano.
} 
(Erdbirnen > peras de tierra). Asimismo, en algunos casos también se observa (8) la combinación de varias técnicas. Los resultados indican que las técnicas predominantes en el corpus son la generalización y la descripción, que tienen un carácter neutralizador. Así, en general, las traducciones analizadas en el estudio de Oster y Molés-Cases (2016) tampoco están muy marcadas culturalmente.

Marco (2019) también analiza las referencias culturales relacionadas con la comida y la bebida, concretamente en el subcomponente inglés-catalán del corpus COVALT. Se basa en una clasificación previa de técnicas de traducción de los referentes culturales (Marco, 2004, 2007), según la cual las técnicas de traducción se organizan en función de dos criterios: la distancia con respecto al lector meta y el grado de intervención del traductor. Distingue tres tipos de técnicas: extranjerizantes, neutralizadoras y familiarizantes. De entre las técnicas extranjerizantes, Marco identifica las siguientes: (1) préstamo puro (cheddar cheese $>$ formatge cheddar), (2) préstamo naturalizado (pudding > púding) y (3) traducción literal (fish and chips $>$ creïlles fregides $i$ peix); en el plano de las técnicas neutralizadoras, se observan las que siguen: (4) descripción (a scoth egg > un ou bullit amb una llonganissa), (5) generalización (ginger ale $>$ refresc), (6) particularización, ${ }^{4}$ (7) amplificación (Leghorn hens $>$ gallines blanques de raça selecta) y su contrapartida, (8) compresión; de entre las técnicas familiarizantes, observa (9) adaptación intracultural (Ginger Nut > Closca de Gingebre), (10) adaptación intercultural (dougnuts > bunyols) y (11) omisión (grog "bebida alcohólica caliente"> -). Este autor también identifica (12) la combinación de técnicas. Los resultados del estudio indican que las técnicas neutralizadoras tienen más presencia que las extranjerizantes y familiarizantes, en este orden. Además, la originalidad del trabajo de Marco (2019) radica en que también hace referencia a los factores que condicionan la elección de las técnicas, siendo los más prominentes los siguientes: la inexistencia del referente del texto origen en la cultura meta, los diferentes grados de institucionalización (cultural markedness), la importación del referente origen a la cultura meta y los diferentes grados de granularidad.

\footnotetext{
${ }^{4}$ Marco (2019) no ejemplifica esta técnica (contraria a la generalización), dado que aparece tan solo en dos ocasiones en el corpus analizado.
} 
De las contribuciones que acabamos de revisar se desprende que, en relación con los referentes culturales de la gastronomía, las traducciones de textos turísticos están bastante más marcadas culturalmente que las de los textos literarios. Este hecho estaría en consonancia con el papel vocativo/apelativo de las traducciones del ámbito turístico al que se refieren Martínez Sánchez e Íñigo Ros (1998), es decir, que el uso de préstamos y otras técnicas extranjerizantes transmite un "color local" interesante desde el punto de vista de la promoción turística.

\section{Metodología}

En el presente trabajo se analizan las páginas web oficiales de las 15 ciudades españolas que han sido reconocidas Ciudad Patrimonio de la Humanidad por la UNESCO, siguiendo los protocolos de la Convención de 1972 para la Protección del Patrimonio Mundial Cultural y Natural. Los estados firmantes de la Convención se reconocen en la obligación de "identificar, proteger, conservar, rehabilitar y transmitir a las generaciones futuras el patrimonio cultural y natural situado en su territorio" (UNESCO, 1972). Las ciudades que tienen este reconocimiento en el estado español son, por orden alfabético: Alcalá de Henares, Ávila, Baeza, Cáceres, Córdoba, Cuenca, Ibiza, Mérida, Salamanca, San Cristóbal de la Laguna, Santiago de Compostela, Segovia, Tarragona, Toledo y Úbeda.

Se utiliza un método mixto de investigación (Dörnyei, 2007; Sandelowsky, 2003), puesto que, más que poblar mundos divergentes, el análisis cuantitativo y cualitativo de los datos se complementa y sirve para ofrecer una visión general y más completa de los mismos, aportando diferentes miradas sobre un mismo corpus.

\subsection{Corpus: selección y recopilación de datos}

El corpus utilizado incluye páginas web en español y sus versiones (traducciones) al inglés. Se compone de los contenidos de las secciones relacionadas con algunos referentes culturales, en concreto con las secciones destinadas a la gastronomía de las páginas web oficiales de las ciudades mencionadas más arriba. Cada Ciudad Patrimonio de la Humanidad tiene su propia página web, a veces vinculada a la del ayuntamiento, otras a la comunidad autónoma. En la Tabla 1 se pueden identificar las páginas analizadas: 


\begin{tabular}{|c|c|c|}
\hline CIUDAD & WEB EN ESPAÑOL & WEB EN INGLÉS \\
\hline $\begin{array}{l}\text { Alcalá de } \\
\text { Henares }\end{array}$ & http://www.turismoalcala.es/ & $\begin{array}{l}\text { http://www.turismoalcala.es/\#!/gastro- } \\
\text { nomia }\end{array}$ \\
\hline Ávila & http://www.avilaturismo.com/es/ & http://www.avilaturismo.com/en/ \\
\hline Cáceres & $\begin{array}{l}\text { http://www.viajarporextremadura. } \\
\text { com/cubic/ap/cubic.php/doc/Ca- } \\
\text { ceres--la-ciudad-medieval-115.htm }\end{array}$ & - \\
\hline Córdoba & $\begin{array}{l}\text { https://www.turismodecordoba. } \\
\text { org/donde-comer }\end{array}$ & $\begin{array}{l}\text { https://www.turismodecordoba.org/ } \\
\text { where-to-eat }\end{array}$ \\
\hline Cuenca & $\begin{array}{l}\text { http://turismo.cuenca.es/portal/ } \\
\text { lang_es-ES/tabid__9395/default. } \\
\text { aspx }\end{array}$ & $\begin{array}{l}\text { https://turismo.cuenca.es/portal/lang } \\
\text { en/tabid_9395/default.aspx }\end{array}$ \\
\hline Ibiza & $\begin{array}{l}\text { http://ibiza.travel/conoce-ibiza/ } \\
\text { vino-y-gastronomia/ }\end{array}$ & $\begin{array}{l}\text { http://ibiza.travel/en/know-ibiza/wine- } \\
\text { and-gastronomy/ }\end{array}$ \\
\hline Mérida & $\begin{array}{l}\text { http://turismomerida.org/donde- } \\
\text { comer/ }\end{array}$ & http://turismomerida.org/where-to-eat \\
\hline Salamanca & $\begin{array}{l}\text { https://www.salamanca.es/es/ } \\
\text { propuestas-especiales/salamanca- } \\
\text { para-comersela }\end{array}$ & https://www.salamanca.es/en/ \\
\hline $\begin{array}{l}\text { San Cristóbal } \\
\text { de la Laguna }\end{array}$ & $\begin{array}{l}\text { https://www.webtenerife.com/ } \\
\text { tenerife/la-isla/municipios/ } \\
\text { laguna/?tab=1 }\end{array}$ & $\begin{array}{l}\text { https://www.webtenerife.co.uk/tenerife/ } \\
\text { the-island/municipalities/laguna/? } \\
\text { ga=2.91277338.1423290431. } \\
1519216775-1562771069 . \\
1519216775\end{array}$ \\
\hline $\begin{array}{l}\text { Santiago de } \\
\text { Compostela }\end{array}$ & http://www.santiagoturismo.com & http://www.santiagoturismo.com \\
\hline Segovia & $\begin{array}{l}\text { http://www.segoviaturismo.es/ } \\
\text { ven-a-segovia/gastronomia-y- } \\
\text { enoturismo/cocina-tradicional }\end{array}$ & $\begin{array}{l}\text { http://www.segoviaturismo.es/en/ } \\
\text { http://visitsegovia.turismodesegovia. } \\
\text { com }\end{array}$ \\
\hline Tarragona & $\begin{array}{l}\text { https://www.tarragonaturisme.cat/ } \\
\text { ca/amb-gust-tarragona }\end{array}$ & https://www.tarragonaturisme.cat/en \\
\hline Toledo & $\begin{array}{l}\text { http://www.turismocastillalaman- } \\
\text { cha.es/gastronomia/lo-tienes-que- } \\
\text { probar/ }\end{array}$ & $\begin{array}{l}\text { http://en.www.turismocastillalamancha. } \\
\text { es/gastronomia/ }\end{array}$ \\
\hline Úbeda & $\begin{array}{l}\text { http://www.turismodeubeda.com/ } \\
\text { index.php/es/conoce-ubeda/guia- } \\
\text { virtual/gastronomia }\end{array}$ & $\begin{array}{l}\text { http://www.turismodeubeda.com/index. } \\
\text { php/en/home }\end{array}$ \\
\hline Baeza & $\begin{array}{l}\text { http://ubedaybaezaturismo.com/ } \\
\text { nuestra-gastronomia/ }\end{array}$ & $\begin{array}{l}\text { http://ubedaybaezaturismo.com/en/our- } \\
\text { gastronomy/ }\end{array}$ \\
\hline
\end{tabular}

Tabla 1. Páginas web utilizadas 
Como se observa en la tabla, hay disparidad en el grado de especificidad de las páginas analizadas. En algunos casos (Santiago de Compostela), no existe una página específica sobre gastronomía, en otros (Cáceres), no aparece una página concreta en inglés. Para el análisis se han intentado identificar los aspectos culturales reflejados en las páginas web relacionados con la comida y la bebida asociadas a las ciudades mencionadas, que se recomiendan en estas páginas web de presentación como identificadores de parte del patrimonio cultural.

Los textos fueron recopilados y analizados a lo largo de 2018. El objeto de este estudio es hacer una extrapolación de las tendencias y las prácticas de traducción más habituales a partir de los datos publicados hasta dicha fecha ${ }^{5}$.

\subsection{Caracterización y particularidades}

La recopilación del corpus fue manual. En algunos casos, las páginas estaban protegidas y hubo que copiarlas como imágenes, lo que hizo imposible su tratamiento o análisis por medios electrónicos o mediante software, de manera que el manejo y estudio de los textos fue manual en su integridad. Los textos ofrecen también otras diferencias y peculiaridades, como por ejemplo la extensión de los escritos en las diferentes ciudades, o el tono y el grado de especificidad elegido en los textos.

Sin embargo, en todas las páginas web, incluso en aquellas de las ciudades en que no había páginas específicas destinadas a la gastronomía, había términos culturales referidos a la gastronomía (ítems relacionados con comida y bebida). En total, se han analizado 431 referencias culturales de esta temática, con el objeto de investigar la tendencia de traducción utilizada.

\subsection{Clasificación de las técnicas utilizadas}

El análisis de los términos identificados en el corpus se ha basado, principalmente, en las clasificaciones presentadas anteriormente en el apartado de fundamentos teóricos, que se han adaptado al estudio para dar cuenta de todos los casos encontrados en el corpus. La clasificación se

\footnotetext{
${ }^{5}$ Debido a la continua actualización de los contenidos, las páginas web podrían haber cambiado total o parcialmente desde entonces.
} 
basa en la consideración de si las técnicas utilizadas en la traducción muestran un propósito de distanciamiento con respecto al destinatario o extranjerización, de acercamiento al lector meta o familiarización, o si son técnicas de neutralización, que sustituyen el término origen por una descripción o explicación más o menos detallada y, por tanto, el referente cultural deja de ser un referente en la cultura meta (cf. Marco, 2019). Además, para analizar las técnicas combinadas, se han tenido en cuenta los casos en los que en la traducción se utiliza más de una técnica. La Tabla 2, más abajo, ayuda a visualizar las diferentes técnicas que se han observado en el análisis:

\begin{tabular}{|l|l|l|}
\hline \multicolumn{1}{|c|}{ Extranjerización } & \multicolumn{1}{c|}{ Neutralización } & \multicolumn{1}{c|}{ Familiarización } \\
\hline Préstamo puro & Descripción & Adaptación intercultural \\
\hline Préstamo naturalizado & Generalización & Adaptación intracultural \\
\hline Traducción literal & Particularización & Omisión \\
\hline
\end{tabular}

Tabla 2. Clasificación de las técnicas utilizadas en las traducciones

\section{Resultados y discusión}

\subsection{Análisis cualitativo}

En este apartado se presentan los resultados cualitativos del estudio, es decir, se ejemplifican las diferentes técnicas de traducción observadas en el análisis, que se presentarán a partir de la información incluida en la Tabla 2.

En primer lugar, se analizan las técnicas de extranjerización (las más utilizadas). Se trata de casos de traducción literal, como en el caso de puchero canario traducido por Canarian stew, o papas arrugadas traducidas por wrinkly potatoes, préstamo puro (morcón traducido por morcón) y préstamo naturalizado (ochio traducido por ochio).

Continuaremos la explicación del análisis a partir de las técnicas de neutralización. Un ejemplo de descripción sería la traducción de la pimienta palmera canaria por local variety of red peppers. Un ejemplo de generalización sería la traducción del mojo canario por un simple good sauce. La particularización, siempre en combinación con otras 
técnicas, se ha visto, por ejemplo, en el caso de empanada traducida por tuna fish pasties, combinando las técnicas de adaptación intercultural y particularización.

En último lugar mencionaremos ejemplos de técnicas de familiarización. Por ejemplo, se ha clasificado como adaptación intercultural el caso de jamón (Figura 1, margen superior izquierdo) traducido por ham (Figura 1, margen inferior izquierdo), o el caso de salchichones (Figura 1, margen superior derecho) traducidos por sausages (Figura 1, margen inferior derecho).
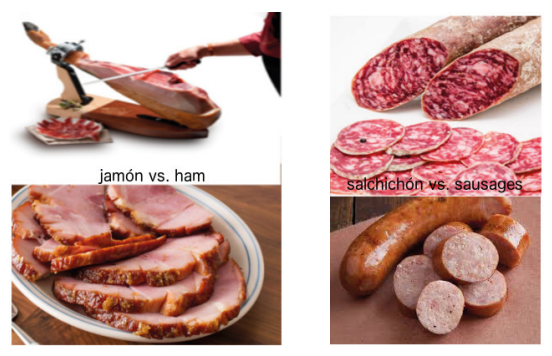

Figura 1. Ejemplos de adaptación intercultural: jamón traducido por ham y salchichones, por sausages

Entre los ejemplos de adaptación intracultural cabría mencionar el caso de ligá traducida por tapa, o tapeo. Las omisiones que se han encontrado en las traducciones son, por ejemplo, calandrajos, o tocinillo de cielo, totalmente excluidos de los textos meta. ${ }^{6}$

Asimismo, un ejemplo de técnicas combinadas es el préstamo naturalizado sumado a descripción del original ochio convertido en el texto meta en sweet or salted buns called ochios.

En el análisis cualitativo se han observado otros fenómenos coincidentes con lo que Pierini (2007) considera resúmenes, textos nuevos parafraseados y no traducciones. Además, en un número considerable de casos, se han encontrado errores de traducción (cf. Soto Almela, 2013), como por ejemplo yemas de Ávila traducidas por yolks, o ciegas (bizcochos segovianos) traducidas por blinds.

\footnotetext{
${ }^{6}$ En estos casos, además, las páginas web no incluyen imágenes, que podrían ejercer un papel compensador.
} 


\subsection{Análisis cuantitativo}

El análisis cuantitativo de los referentes culturales, en concreto de términos relacionados con la gastronomía, muestra que las técnicas de extranjerización sobresalen de manera muy significativa sobre el resto de técnicas. De los 487 casos analizados, 368 presentan esta tendencia, mientras que en 62 se observan técnicas de familiarización. Solamente en 51 de los casos los traductores han optado por técnicas de neutralización. Asimismo, 6 casos se han clasificado como "otros", para dar cuenta de errores.

En el gráfico que figura a continuación (Figura 2) se puede observar la distribución de los resultados obtenidos para la totalidad de las técnicas de traducción.

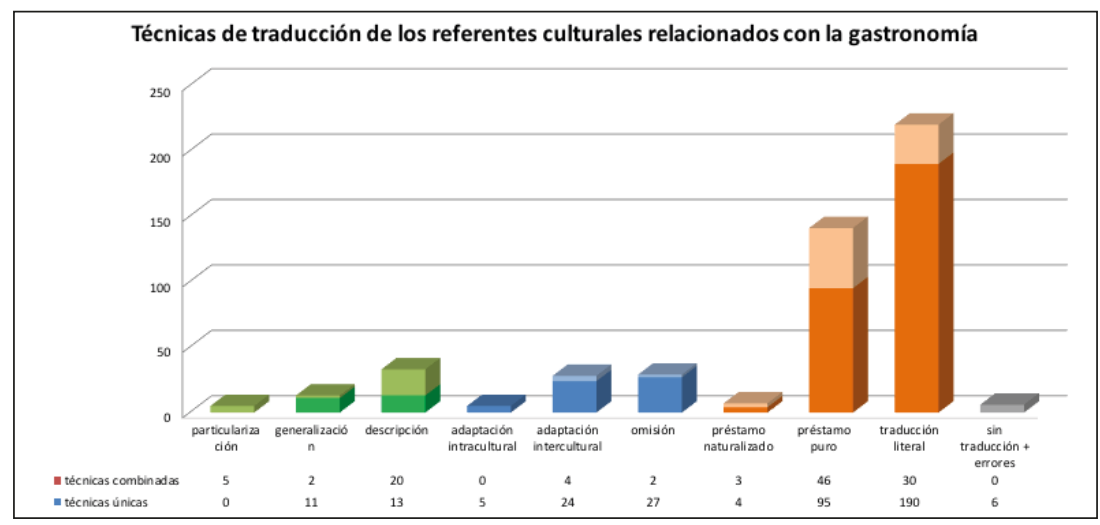

Figura 2. Distribución de las técnicas de traducción.

El gráfico también muestra el número de casos que cada técnica se utiliza en combinación con otras, para dar cuenta de si son técnicas que se utilizan de manera singular o si dependen o se apoyan en otras en las traducciones. Cabe mencionar también que la particularización, incluida entre las técnicas de neutralización, no se da nunca en solitario y siempre aparece en combinación con otras técnicas como la adaptación intercultural y la traducción literal, en un total de 24 ocasiones. 
A continuación miraremos con detenimiento las ocasiones en que las técnicas se usan en combinación con otras, para indagar cuáles son las combinatorias más frecuentes (Figura 3).

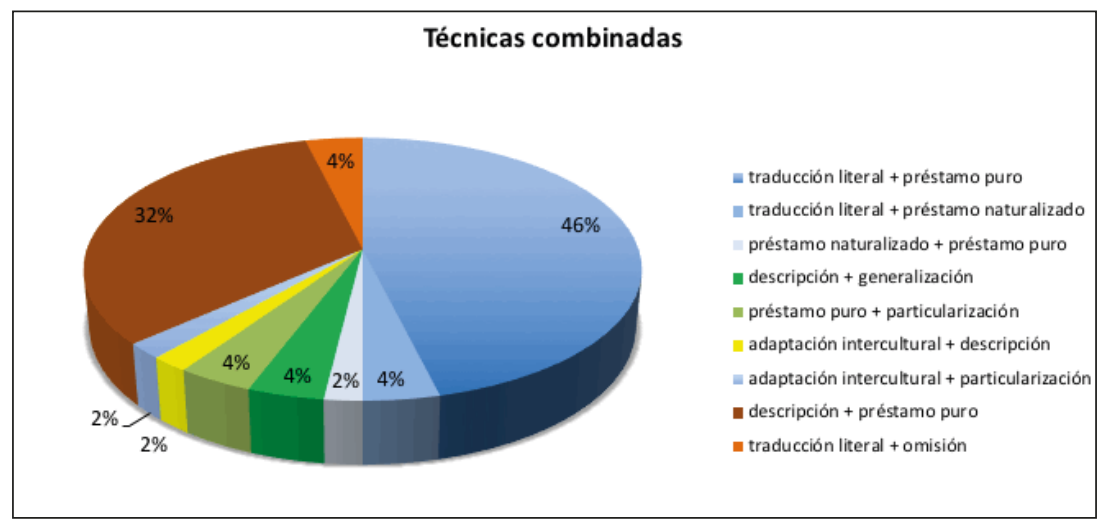

Figura 3. Distribución de las técnicas combinadas.

Así, en el $13 \%$ del total de los casos, es decir en 56 ocasiones, se ha optado por combinar varias técnicas en la traducción de los referentes hacia la lengua meta. Como se puede ver en la Figura 3, hay una gran variedad de combinatorias. La técnica más utilizada en combinación es el préstamo puro. Sobresale sobre todo en combinación con la traducción literal (i. e. manzana reineta del Barco de Ávila traducida por Reineta apples from El Barco de Ávila), que se da en el $43 \%$ de los casos de combinación, o la de descripción unida al préstamo puro (30\% de los casos de combinación). Un ejemplo de esta combinación de técnicas sería la traducción de ajoblanco por ajoblanco (typical southern-style cold soup).

En el caso de las técnicas de familiarización, la omisión es la más utilizada ( 29 casos, 2 de los cuales en combinación con otras técnicas), seguida de cerca por la adaptación intercultural ( 28 casos, 4 de los cuales en combinación). Un ejemplo del primer caso podría ser variedad de cornezuelo, ausente en el texto meta, un ejemplo del segundo sería la traducción de turrones por nougats.

En lo referente a las técnicas de neutralización, la distribución se reparte entre la descripción (33 casos en total), como en cochino negro, 
una especie autóctona traducido por pork obtained from the native species of black pigs y la generalización (13 casos), como en conservas de lomo, cerdo, morcilla en aceite, que aparece traducido por meat preserves.

Las técnicas de extranjerización tienen una distribución también desigual, en la que la gran mayoría de los casos que utilizan esta estrategia $(66 \%)$ se utiliza la traducción literal, como por ejemplo aceitunas machadas traducido por crushed olives. El préstamo puro, con el $33 \%$ de los casos, también está muy presente en las traducciones, en las que se opta por dejar el término en lengua origen: morcón por morcón.

Por último, la Figura 4 recoge la información anterior clasificada según la estrategia/tendencia de traducción.

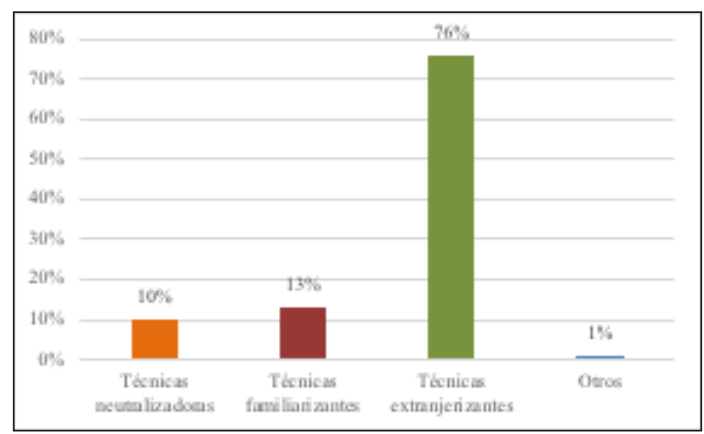

Figura 4. Estrategias de extranjerización, naturalización y familiarización.

Tal y como se desprende de los resultados, en la gran mayoría de los casos se ha optado por técnicas de extranjerización en las traducciones, que alejan el texto de la cultura meta y lo mantienen cercano a la cultura origen. Estos datos son coherentes con los presentados por estudios previos de temática similar (cf. Martínez Sánchez e Íñigo Ros, 1998; Rodríguez Abella, 2009).

\section{Conclusiones}

La traducción ejerce un papel clave en la transferencia cultural y, por tanto, recurrir a la traducción especializada es de gran importancia en 
términos de promoción turística, donde no deberían caber los casos de traducción automática sin posedición, sino la traducción profesional realizada por un agente humano especializado en el tema. Asimismo, la designación de un lugar como Patrimonio Cultural Inmaterial de la $\mathrm{Hu}-$ manidad de la UNESCO le concede a dicho lugar un estatus de reconocimiento. Y para mantener dicho estatus y credibilidad, estas ciudades deben cumplir con una serie de obligaciones, que incluyen no solamente cuidar sus casos históricos y entorno, sino también la puesta en valor y publicación de su patrimonio cultural a partir de folletos y páginas web traducidas por profesionales, ya que las traducciones malas o erróneas no hacen sino dañar la imagen que la ciudad proyecta al mundo. Este hecho es especialmente importante en un país como España, donde el turismo es uno de los grandes motores de la economía.

Por último, y no por ello menos importante, sigue siendo necesario resaltar la importancia de la traducción profesional en este sector, no solo en el marco de los estudios en Traducción e Interpretación a partir de la impartición de asignaturas enfocadas concretamente a la traducción del campo del turismo y sus particularidades (cf. Durán Muñoz, 2012), sino también en el contexto del grado en Turismo, a partir de prácticas de aula (relacionadas, por ejemplo, con la lengua extranjera, la lengua para fines específicos y la comunicación intercultural) que conciencien al colectivo de los profesionales del sector del Turismo sobre la importancia de la traducción profesional en este sector y que les lleve a valorar el impacto de las traducciones de calidad.

\section{Bibliografía}

Chesterman, Andrew. 1997. Memes of translation. Amsterdam: John Benjamins.

Dörnyei, Zoltan. 2007. Research Methods in Applied Linguistics. Oxford: Oxford University Press.

Durán Muñoz, Isabel. 2012. Caracterización de la traducción turística: problemas, dificultades. Revista de Lingüística y Lenguas Aplicadas 7: 103113. doi: 10.4995/rlyla.2012.1127

Kelly, Dorothy. 1998. The translation of texts from the tourist sector. Trans 2: 33-42. doi: http://dx.doi.org/10.24310/TRANS.1998.v0i2.2354

Marco, Josep. 2004. Les tècniques de traducció (dels referents culturals): retorn per a quedar-nos-hi. Quaderns. Revista de traducció 11, 129-149. 
Marco, Josep. 2007. The terminology of translation: Epistemological, conceptual and intercultural problems and their social consequences. Target 19(2): 255-269. doi: https://doi.org/10.1075/target.19.2.06mar

Marco, Josep. 2019. The translation of food-related culture-specific ítems in the Valencian Corpus of Translated Literature (COVALT) corpus: a study of techniques and factors. Perspectives 27(1): 20-41. doi: https:// doi.org/10.1080/0907676X.2018.1449228

Martínez Sánchez, Patricia C. e Íñigo Ros, Marta. 1998. La traducción de términos culturales en los folletos turísticos: la gastronomía. Quaderns de filología 4(1): 153-170.

Molina, Lucía \& Hurtado, Amparo. 2002. Translation Techniques Revisited: A Dynamic and Functionalist Approach. Meta 47(4): 498-512.

Neubert, Albrecht \& Gregory M. Shreve. 1992. Translation as Text. Kent/London: The Kent State University Press.

Newmark, Peter. 1988. A Textbook of Translation. Nueva York: Prentice-Hall International.

Oster, Ulrike \& Molés Cases, Teresa. 2016. Eating and drinking seen through translation: A study of food-related translation difficulties and techniques in a parallel corpus of literary texts. Across Languages and Cultures 17(1), 53-75. doi: 10.1556/084.2016.17.1.3

Pierini, Patrizia. 2007. Quality in Web Translation: An investigation into UK and Italian Tourism Web Sites. The Journal of Specialized Translators 8: 85-103.

Rodríguez Abella, Rosa M. 2008. El hombre de mi vida: análisis de la traducción de los culturemas del ámbito gastronómico. En Navarro, Carmen, Rodriguez Abella, Rosa M., Dalle Pezze. Francesa \& Miotti, Renzo (eds.) La comunicación especializada. Frankfurt am Main: Peter Lang, 319-355.

Rodríguez Abella, Rosa M. 2009. La traducción de los culturemas en el ámbito de la gastronomía (Análisis de los folletos de TURESPAÑA). EUT Edizioni Università di Trieste, 47-69.

Sandelowski, Margarete. 2003. Tables or tableaux? The challenges of writing and reading mixed methods studies. En Tashakkori, Abbas \& Teddlie, Charles (eds.) Handbook of mixed methods in social \& behavioral research. Sage: Thousand Oaks, 321-350.

Soto Almela, Jorge. 2013. La traducción de términos culturales en el contexto turístico español-inglés: recepción real en usuarios anglófonos. Quaderns 20: 235-250.

Toury, Gideon. 1995. Descriptive Translation Studies and Beyond. Ámsterdam/Filadelfia: John Benjamins. 
UNESCO. 1972. Convención sobre la protección del patrimonio mundial, cultural y natural. https://es.unesco.org/themes/patrimonio-mundial. [Acceso: 15/03/2019].

Venuti, Lawrence. 1995. The Translator's Invisibility. Londres: Routledge. 